\title{
Land use/land cover classification using machine learning models
}

\author{
Subhra Swetanisha1, Amiya Ranjan Panda1, Dayal Kumar Behera² \\ ${ }^{1}$ School of Computer Engineering, KIIT Deemed to be University, Bhubaneswar, India \\ ${ }^{2}$ Faculty of Computer Science and Engineering, Silicon Institute of Technology, Bhubaneswar, India
}

\begin{tabular}{l} 
Article Info \\
\hline Article history: \\
Received Apr 28, 2021 \\
Revised Sep 20, 2021 \\
Accepted Oct 10, 2021 \\
\hline Keywords: \\
Land use and land cover \\
Machine learning \\
Random forest \\
Remote sensing \\
Support vector machine \\
XGBoost
\end{tabular}

\section{Corresponding Author:}

Dayal Kumar Behera

Department of CSE, Silicon Institute of Technology

Bhubaneswar, India

Email: dayalbehera@gmail.com

\begin{abstract}
An ensemble model has been proposed in this work by combining the extreme gradient boosting classification (XGBoost) model with support vector machine (SVM) for land use and land cover classification (LULCC). We have used the multispectral Landsat- 8 operational land imager sensor (OLI) data with six spectral bands in the electromagnetic spectrum (EM). The area of study is the administrative boundary of the twin cities of Odisha. Data collected in 2020 is classified into seven land use classes/labels: river, canal, pond, forest, urban, agricultural land, and sand. Comparative assessments of the results of ten machine learning models are accomplished by computing the overall accuracy, kappa coefficient, producer accuracy and user accuracy. An ensemble classifier model makes the classification more precise than the other state-of-the-art machine learning classifiers.
\end{abstract}

This is an open access article under the CC BY-SA license.

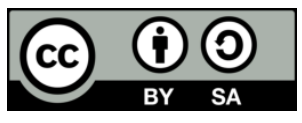

\section{INTRODUCTION}

Land use and land cover classification (LULCC) is a critical technique for assessing global change at various spatiotemporal scales [1]. It is a pervasive, accelerating, and substantial process fueled by human activity and frequently results in changes that directly affect humans. The effects of LULCC on ecosystem sustainability are becoming a growing focus of global change study [2]. Till today, there has been a requirement to deliver provincial land use and land cover (LULC) maps and information for a variety of purposes, including change detection [3], planning or monitoring of the urban environment [4], disaster monitoring, landscape planning, resource management, site suitability analysis and ecological studies [5] or biological investigation [6]. Traditionally, non-parametric machine-learning classifiers (ML) such as random forests (RF) and support vector machines (SVMs) [7] have been used for geographical and easy-to-use classification.

The focus of this work is to identify the physical aspect of the earth's surface (land cover) as well as how we exploit the land (land use) for the twin cities of Odisha. This can be accomplished by field surveys or through the analysis of satellite pictures (remote sensing) [6]. Conducting field surveys is more thorough and authoritative. It is a costly endeavor that frequently takes a long time to complete. But with recent advancements in the space sector and an increase in the availability of satellite photos (both free and commercial), machine learning models [8] have demonstrated promising outcomes in this field. Recent advancements in sensor technology have resulted in the development of a constellation of satellites [9] and airborne platforms from which a significant amount of spatial resolution remotely sensed imagery is available. Landsat-8 [10] is now circling the earth. The operational land imager sensor (OLI) offers images in 
six distinct spectral bands on the Landsat payload. In this paper, the data of Landsat-8 is used for classification. The contributions of the work are i) land use and land cover classification using machine learning models; ii) generating the feature set from the raster using the shapefile of the training and test data; iii) designing an ensemble model by combining the output of the XGBoost model with SVM; and iv) performing efficacy analysis of ensemble models in view of user, producer, and overall accuracy.

\section{LITERATURE SURVEY}

Many works have been done to examine the use of LULC analysis on remotely sensed records. From 1986 to 2001 in Pallisa District, Uganda, Otukei and Blaschke [3] carried out land cover mapping and land cover assessing using DTs, SVMs and MLCs. They explored the use of knowledge mining to find the required classification bands and thresholds for decision. The analysis assessed the efficiency of the classification models, claiming that land cover elements occur at an unpredictable pace.

According to desired classes, a few image classification models are available for segmenting a multi-dimensional component space into homogenous regions and labelling segments. Parametric classifiers accept a normally distributed dataset and statistical parameters acquired properly from training data. The most broadly utilized parametric classifier is the maximum-likelihood classifier (MLC), which makes decision surfaces dependent on the mean and covariance of each class. MLC [11] was first applied to IRS LISS-III images between 2001 and 2011 and classified into eight classes. Additionally, the study used a unique methodological framework for post-classification adjustments. It considerably increased total classification accuracy from $67.84 \%$ to $82.75 \%$ in 2001 and from $71.93 \%$ to $87.43 \%$ in 2011 .

Islam et al. [1] used Landsat TM and Landsat 8 OLI/TIRS images to examine land use changes in Chunati Wildlife Sanctuary (CWS) from 2005 to 2015. ArcGIS and ERDAS imagine were used for land use change assessment. To derive supervised land use categorization, the maximum likelihood classification technique was applied. It was discovered that around 256 ha of the degraded forest area has increased over ten years (2005-2015), with an annual rate of change of 25.56\%. Non-parametric classifiers do not accept a particular information appropriation to isolate a multi-dimensional feature space into classes. Most normally utilized non-parametric classifiers incorporate decision trees [4], support vector machines (SVM) [12] and expert systems.

ML algorithms have been utilized according to pixel classifiers in remote sensing image analysis [6]. Grippa et al. [13] describes a method for mapping urban land use at the street block level, emphasizing residential usage by utilizing very-high-resolution satellite images and derived land-cover maps as input. For the classification of street blocks, a random forest (RF) classifier is utilized, which achieves accuracies of $84 \%$ and $79 \%$ for five and six land-use classifications, respectively. RF classifier applied over urban communities Dakar and Ouagadougou, cover more than $1,000 \mathrm{~km}^{2}$ altogether, with a spatial resolution of $0.5 \mathrm{~m}$. In the year 2019, Jamali [7] compared and contrasted eight machine learning methods for image categorization in the northern region of Iran developed in the Waikato environment for knowledge analysis (WEKA) and R programming languages. Machine learning models [14]-[16] such as RF, SVM [17], [18], decision tree, K-nearest-neighbors (KNN) [19], principal component analysis (PCA) [20] are successfully applied in many application areas. We have built up an ensemble model [21], including SVM and XGBoost [22], that gives better precision when contrasted with other individual machine learning models.

\section{LULC CLASSIFICATION}

\subsection{Study area}

Our study site is the twin cities of Odisha i.e., Bhubaneswar and Cuttack, which are situated towards the Eastern part and lies between $20^{\circ} 15^{\prime} \mathrm{N}-20^{\circ} 28^{\prime} \mathrm{N}$ latitude and $85^{\circ} 52^{\prime} \mathrm{E}-85^{\circ} 54^{\prime} \mathrm{E}$ longitude. According to the European petroleum survey group (EPSG), the twin cities of Odisha lies in EPSG:32645-WGS $84 /$ UTM zone $45 \mathrm{~N}$. It is surrounded by Ganjam district towards the north, Puri, Jagatsinghpur and Kendrapara districts towards the east, Jajpur and Dhenkanal districts towards the south and Anugul, Buodh and Nayagarh districts to the west. Bhubaneswar is the capital of Odisha, coming under the Khurda district. The urban administrative area of the twin cities is considered for analysis.

\subsection{Data acquisition}

To establish land usage, land cover (LU/LC) of the study area, Landsat satellite-8 ETM+data for 2020 have been used. The spectrum consists of six electromagnetic (EM), shortwave Infra-Red1-SWER 1 and shortwave Infra-Red2-SWIR 2 including blue, green, red, near infra-red, which is used to classify into seven land use classes such as a river, canal, pond, forest, urban, agricultural land, sand. 


\subsection{Image pre-processing}

\subsubsection{Layer stacking}

After data acquisition, this stage is to apply fundamental pre-processing activities framed on the raw Satellite images before its utilization in any further upgrade, understanding, interpretation or analysis. Layer stacking [23] is applied to consolidate various images into a single image. The LULC map after layer stacking is portrayed in Figure 1(a).

\subsubsection{Atmospheric correction}

The atmospheric correction [1] is essential when working on images with more than one timestamp. We not only implement image classification but also want to compare several images between one another. The main aim is the conversion of raster bands of Landsat 8 images from digital numbers to reflectance. Atmospheric correction is applied on the resultant image of layer stacking shown in Figure 1(a). In our work, the atmospheric correction is implemented using the dark object subtraction method. After implementing atmospheric correction, the generated map is shown in Figure 1(b).

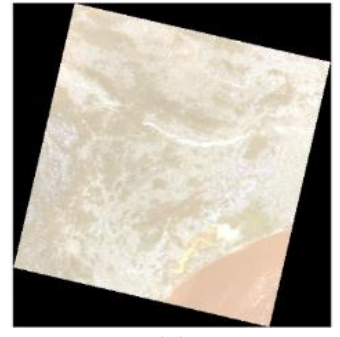

(a)

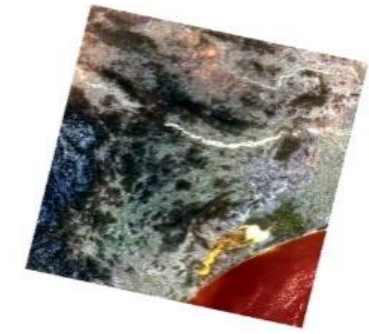

(b)

Figure 1. Image pre-processing (a) after layer stacking and (b) after atmospheric correction

\subsubsection{Image composite}

Each band of a multispectral image can be shown each band as a grayscale image or as a mix of three bands simultaneously as a color composite image. The three essential shades of light are red, green, and blue (RGB). PC screens can show a picture of three unique groups by utilizing an alternate essential color for each band. When we consolidate these three images, the outcome is a color image with every pixel's shading controlled by a mix of RGB of various splendors. Our study area is cropped from the LULC map shown in Figure 1. Two different color composite formats: True color composite and false color composite [24] of the study area is depicted in Figures 2(a) and 2(b), respectively.

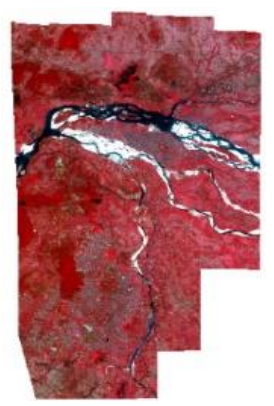

(a)

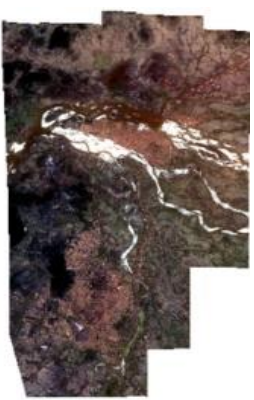

(b)

Figure 2. Image composite of twin cities of Odisha in (a) false color and (b) true color

\section{METHOD}

The proposed classification method consists of three major stages. Firstly, the study area is identified, and data acquisition is performed. In the next stage, image pre-processing is carried out with layer stacking and atmospheric correction. Finally, ensemble classification is carried out for thematic LULC change analysis. 
Image classification is an automated approach for classifying raster data belongs to satellite images [25], [26], airborne images, and drone images. This typically includes evaluating several images and applying statistical rules in determining the identity of the land cover for each pixel in an image. In this paper, supervised classification algorithms are applied for LULC classification. All classes of interest are selected to prepare the train and test dataset. The proposed model is depicted in Figure 3. The training data is used to train the classifier, whereas the test data is used to validate the model. Raster library of $\mathrm{R}$ is used to generate the features from the raster and shapefile of the train and test data. The generated features are taken as input to implement the classifier using Python's sklearn library with default parameter setting. For the ensemble model, voting classifier is used with 0.5 weight for both SVM and XGBoost models.

After defining the classes, the next step is training stage. Here numerous training areas for the required land cover classes are identified. A sufficient sample size is required to ensure accurate statistical descriptors of our training data. The LULC maps generated in different machine learning models are shown in Figure 4. Output maps of minimum distance, RF and Hybrid model (SVM+XGBoost) are shown in Figures 4(a), 4(b), and 4(c), respectively.

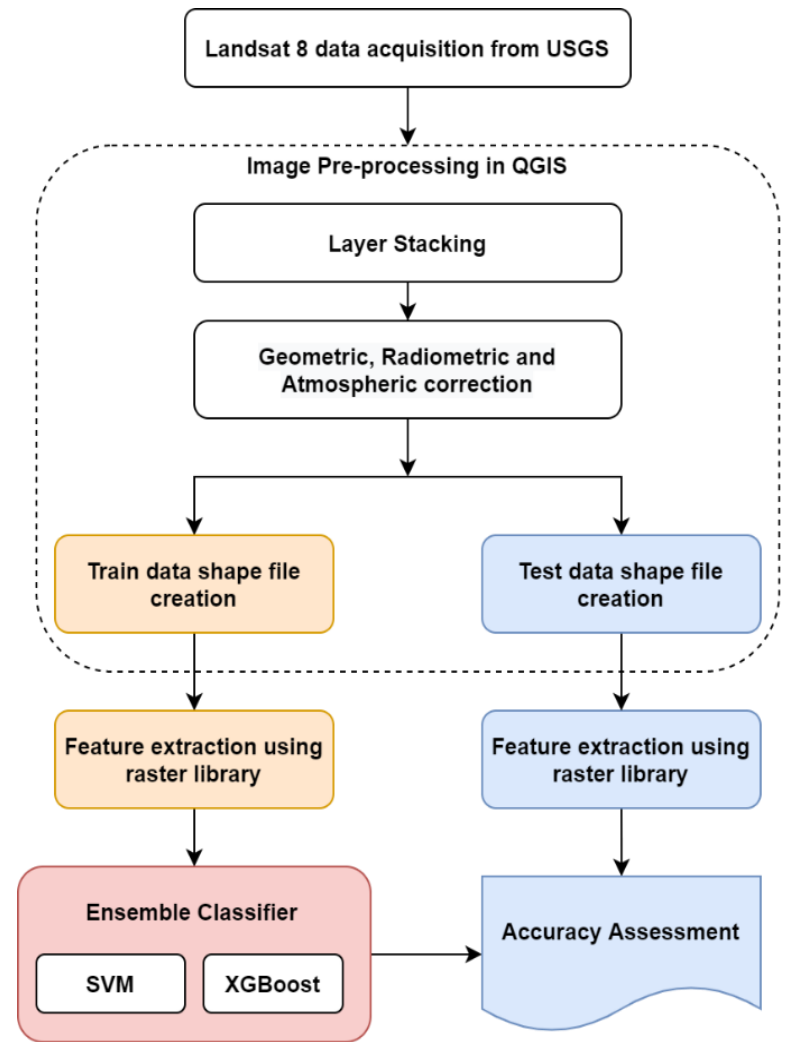

Figure 3. Proposed model

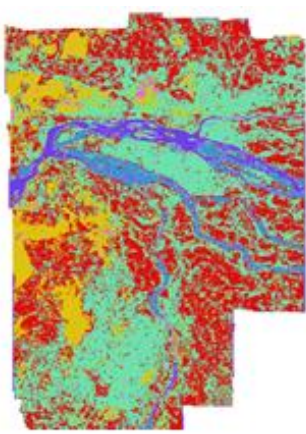

(a)

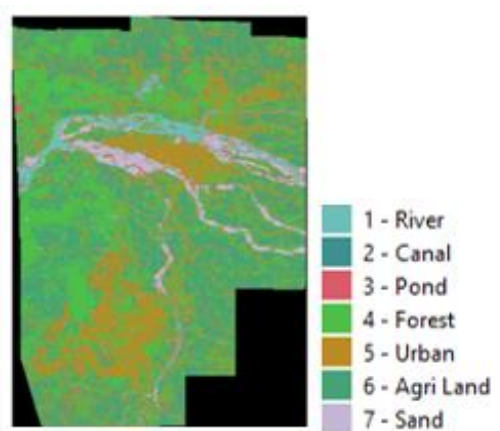

(b)

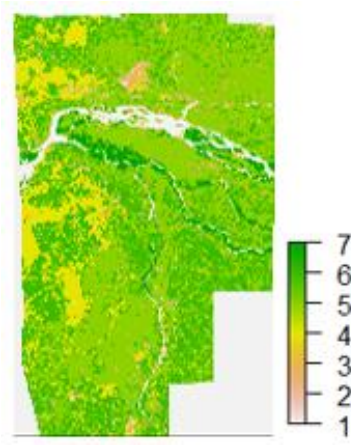

(c)

Figure 4. Classification using (a) minimum distance, (b) random forest (RF), and (c) SVM+XGBoost 


\section{RESULTS AND DISCUSSION}

Then the accuracy assessment is done to determine that how good the map is. If the accuracy assessment shows that the land use land cover map is valid then the resulting map can be utilized in different ways like dramatic maps, all kind of output tables or statistics for the various land cover classes and digital data files amendable to inclusion in geographical information system (GIS). In supervised learning classification, an error occurs when a pixel that belongs to one class is allotted to another class. Here the question is how to test for it. And basically, there are two methods either visual control or quantitative control to test this.

Visual control is basically visual assessment of the results of supervised or unsupervised learning. Once the visual control has passed and if the results look plausible then the quantitative approach for accuracy assessment can be done. Accuracy assessment procedure with the help of error metrics plays vital role in any classification job and plays a vital role in LULC classification. This is done by calculating different accuracy measures like overall accuracy (OA), user accuracy (UA), producer accuracy (PA), Kappa coefficient from the confusion matrix. Table 1 shows a comparison between different Machine learning models based on train accuracy, overall accuracy and Kappa index [27], [28]. Higher value indicates better classification. Table 2 depicts PA and UA of ML classifiers for classifying seven class labels.

Table 1. Model accuracy and Kappa coefficient of ML classifiers

\begin{tabular}{cccc}
\hline Model & Train accuracy & Test accuracy (OA) & Kappa coefficient \\
\hline Minimum distance & 0.8592 & 51.77 & 0.413 \\
KNN & 0.9952 & 93.0782 & 0.8738 \\
LR & 0.9404 & 93.0049 & 0.8682 \\
DT & 1.0000 & 92.4922 & 0.8631 \\
SVM & 0.9514 & 93.3208 & 0.8742 \\
XGBoost & 0.9957 & 93.5360 & 0.8818 \\
Extra tree & 0.9980 & 93.2064 & 0.8760 \\
RF & 0.8776 & 88.6330 & 0.7872 \\
SVM+XGBoost & 0.9920 & 93.5635 & 0.8824 \\
RF+XGBoost & 0.9907 & 93.4902 & 0.8806 \\
\hline
\end{tabular}

Table 2. Producer and UA of ML classifiers

\begin{tabular}{cccccccccccccccc}
\hline Model & \multicolumn{2}{c}{ River } & \multicolumn{2}{c}{ Canal } & \multicolumn{2}{c}{ Pond } & \multicolumn{3}{c}{ Forest } & \multicolumn{3}{c}{ Urban } & \multicolumn{3}{c}{ Agri. Land } \\
& PA & UA & PA & UA & PA & UA & PA & UA & PA & UA & PA & UA & PA & UA \\
\hline KNN & 99.60 & 99.98 & 87.28 & 90.49 & 69.23 & 49.32 & 99.93 & 73.54 & 77.33 & 98.00 & 13.07 & 38.89 & 100 & 99.02 \\
LR & 96.68 & 100 & NaN & 0.00 & NaN & 0.00 & 97.90 & 85.03 & 64.34 & 99.13 & 12.57 & 5.83 & 100 & 99.51 \\
DT & 99.41 & 99.95 & 82.02 & 79.75 & 53.89 & 44.29 & 99.51 & 72.87 & 81.20 & 96.73 & 11.91 & 40.00 & 99.92 & 96.17 \\
SVM & 96.68 & 100 & 0.00 & 0.00 & 0.00 & 0.00 & 98.10 & 85.46 & 69.86 & 98.60 & 23.65 & 21.94 & 100 & 99.59 \\
XGBoost & 99.38 & 99.97 & 76.20 & 82.52 & 62.87 & 47.95 & 100 & 76.60 & 84.14 & 98.00 & 13.13 & 37.78 & 99.92 & 99.51 \\
Extra tree & 99.50 & 99.98 & 84.41 & 88.04 & 79.69 & 46.58 & 99.90 & 74.38 & 80.33 & 98.00 & 12.83 & 39.72 & 100 & 99.35 \\
RF & 98.08 & 99.73 & NaN & 0.00 & 0.00 & 0.00 & 97.21 & 92.53 & 42.28 & 100 & NaN & 0.00 & NaN & 0.00 \\
SVM+ & 99.60 & 99.96 & 82.16 & 86.20 & 62.89 & 45.66 & 100 & 77.16 & 80.31 & 98.73 & 10.54 & 28.33 & 99.92 & 99.51 \\
XGBoost & & & & & & & & & & & & & & & \\
RF+ & 99.13 & 99.97 & 89.05 & 74.85 & 66.67 & 43.84 & 99.82 & 78.54 & 80.06 & 99.07 & 6.95 & 17.50 & 99.92 & 99.51 \\
XGBoost & & & & & & & & & & & & & & \\
\hline
\end{tabular}

\section{CONCLUSION}

Land use and land cover classification is beneficial to explore the change dynamics of the city. Although the maximum likelihood classifier is widely used, it could not perform satisfactorily to ensure the desired classification accuracy. This work presented the pixel-based classification of LULC using various ML models. This will benefit the researcher to recognize the best classifiers and various evaluation metrics. Landsat 8 geospatial data with atmospheric correction significantly improve the accuracy of LULC classification. An ensemble model is proposed by combining the output of SVM and Extreme gradient boosting model. The efficacy of the proposed model is shown in Table 2. It is seen that the user, producer, and overall accuracy has been significantly improved in ensemble models.

\section{REFERENCES}

[1] K. Islam, M. Jashimuddin, B. Nath, and T. K. Nath, "Land use classification and change detection by using multi-temporal remotely sensed imagery: The case of Chunati wildlife sanctuary, Bangladesh," The Egyptian Journal of Remote Sensing and Space Science, vol. 21, no. 1, pp. 37-47, Apr. 2018, doi: 10.1016/j.ejrs.2016.12.005.

[2] C. Kok Yang, F. Pei Shan, and T. Lea Tien, "Climate change detection in Penang Island using deterministic interpolation 
methods," Indonesian Journal of Electrical Engineering and Computer Science (IJEECS), vol. 19, no. 1, pp. 412-419, Jul. 2020, doi: 10.11591/ijeecs.v19.i1.pp412-419.

[3] J. R. Otukei and T. Blaschke, "Land cover change assessment using decision trees, support vector machines and maximum likelihood classification algorithms," International Journal of Applied Earth Observation and Geoinformation, vol. 12, pp. S27S31, Feb. 2010, doi: 10.1016/j.jag.2009.11.002.

[4] L. Hua, X. Zhang, X. Chen, K. Yin, and L. Tang, "A feature-based approach of decision tree classification to map time series urban land use and land cover with Landsat 5 TM and Landsat 8 OLI in a Coastal City, China," ISPRS International Journal of Geo-Information, vol. 6, no. 11, pp. 1-18, Oct. 2017, doi: 10.3390/ijgi6110331.

[5] I. A. Md Zin, Z. Ibrahim, D. Isa, S. Aliman, N. Sabri, and N. N. A. Mangshor, "Herbal plant recognition using deep convolutional neural network," Bulletin of Electrical Engineering and Informatics (BEEI), vol. 9, no. 5, pp. 2198-2205, Oct. 2020, doi: 10.11591/eei.v9i5.2250.

[6] Q. Weng, "Remote sensing of impervious surfaces in the urban areas: Requirements, methods, and trends," Remote Sensing of Environment, vol. 117, pp. 34-49, Feb. 2012, doi: 10.1016/j.rse.2011.02.030.

[7] A. Jamali, "Evaluation and comparison of eight machine learning models in land use/land cover mapping using Landsat 8 OLI: a case study of the northern region of Iran," SN Applied Sciences, vol. 1, no. 11, Nov. 2019, Art. no. 1448, doi: 10.1007/s42452019-1527-8.

[8] D. K. Behera, M. Das, S. Swetanisha, and P. K. Sethy, "Hybrid model for movie recommendation system using content K-nearest neighbors and restricted Boltzmann machine," Indonesian Journal of Electrical Engineering and Computer Science (IJEECS), vol. 23, no. 1, pp. 445-452, Jul. 2021, doi: 10.11591/ijeecs.v23.i1.pp445-452.

[9] A. A. W. Mohammed and H. T. Khamees, "Categorizing and measurement satellite image processing of fire in the forest greece using remote sensing," Indonesian Journal of Electrical Engineering and Computer Science (IJEECS), vol. 21, no. 2, pp. 846-853, Feb. 2021, doi: 10.11591/ijeecs.v21.i2.pp846-853.

[10] Z. Deng, X. Zhu, Q. He, and L. Tang, "Land use/land cover classification using time series Landsat 8 images in a heavily urbanized area," Advances in Space Research, vol. 63, no. 7, pp. 2144-2154, Apr. 2019, doi: 10.1016/j.asr.2018.12.005.

[11] A. K. Thakkar, V. R. Desai, A. Patel, and M. B. Potdar, "Post-classification corrections in improving the classification of land use/land cover of arid region using RS and GIS: The case of Arjuni watershed, Gujarat, India," The Egyptian Journal of Remote Sensing and Space Science, vol. 20, no. 1, pp. 79-89, Jun. 2017, doi: 10.1016/j.ejrs.2016.11.006.

[12] A. V. Rajeswari, S. Saritha, and G. Santhosh Kumar, "Classification based land use/land cover change detection through Landsat images," in 2014 International Conference on Data Science \& Engineering (ICDSE), Aug. 2014, pp. 232-237, doi: 10.1109/ICDSE.2014.6974644.

[13] T. Grippa et al., "Mapping urban land use at street block level using OpenStreetMap, remote sensing data, and spatial metrics," ISPRS International Journal of Geo-Information, vol. 7, no. 7, Jun. 2018, Art. no. 246, doi: 10.3390/ijgi7070246.

[14] W. Hassan, T.-S. Chou, O. Tamer, J. Pickard, P. Appiah-Kubi, and L. Pagliari, "Cloud computing survey on services, enhancements and challenges in the era of machine learning and data science," International Journal of Informatics and Communication Technology (IJ-ICT), vol. 9, no. 2, pp. 117-139, Aug. 2020, doi: 10.11591/ijict.v9i2.pp117-139.

[15] S. M. Praveena, R. Kanmani, and A. K. Kavitha, "A neuro fuzzy image fusion using block based feature level method," International Journal of Informatics and Communication Technology (IJ-ICT), vol. 9, no. 3, pp. 195-204, Dec. 2020, doi: 10.11591/ijict.v9i3.pp195-204.

[16] T. R. S. Mary and S. Sebastian, "Predicting heart ailment in patients with varying number of features using data mining techniques," International Journal of Informatics and Communication Technology (IJ-ICT), vol. 8, no. 1, pp. 56-62, Apr. 2019, doi: 10.11591/ijict.v8i1.pp56-62.

[17] N. Kulkarni, "Support vector machine based alzheimer's disease diagnosis using synchrony features," International Journal of Informatics and Communication Technology (IJ-ICT), vol. 9, no. 1, pp. 57-62, Apr. 2020, doi: 10.11591/ijict.v9i1.pp57-62.

[18] A. M. Abdu, M. M. Mokji, and U. U. Sheikh, "Machine learning for plant disease detection: An investigative comparison between support vector machine and deep learning," IAES International Journal of Artificial Intelligence (IJ-AI), vol. 9, no. 4, pp. 670-683, 2020, doi: 10.11591/ijai.v9.i4.pp670-683.

[19] H. Ohmaid, S. Eddarouich, A. Bourouhou, and M. Timouyas, "Comparison between svm and knn classifiers for iris recognition using a new unsupervised neural approach in segmentation," IAES International Journal of Artificial Intelligence (IJ-AI), vol. 9, no. 3, pp. 429-438, 2020, doi: 10.11591/ijai.v9.i3.pp429-438.

[20] S. M. Shaharudin, N. Ahmad, and S. M. C. M. Nor, "A modified correlation in principal component analysis for torrential rainfall patterns identification," IAES International Journal of Artificial Intelligence (IJ-AI), vol. 9, no. 4, pp. 655-661, Dec. 2020, doi: 10.11591/ijai.v9.i4.pp655-661.

[21] A. Yusuf and A. John, "Classifiers ensemble and synthetic minority oversampling techniques for academic performance prediction," International Journal of Informatics and Communication Technology (IJ-ICT), vol. 8, no. 3, pp. 122-127, Dec. 2019, doi: 10.11591/ijict.v8i3.pp122-127.

[22] D. K. Behera, M. Das, S. Swetanisha, J. Nayak, S. Vimal, and B. Naik, "Follower link prediction using the XGBoost classification model with multiple graph features," Wireless Personal Communications, Apr. 2021, doi: 10.1007/s11277-02108399-y.

[23] A. Hailu, S. Mammo, and M. Kidane, "Dynamics of land use, land cover change trend and its drivers in Jimma Geneti District, Western Ethiopia," Land Use Policy, vol. 99, Dec. 2020, Art. no. 105011, doi: 10.1016/j.landusepol.2020.105011.

[24] T. Mugiraneza, Y. Ban, and J. Haas, "Urban land cover dynamics and their impact on ecosystem services in Kigali, Rwanda using multi-temporal Landsat data," Remote Sensing Applications: Society and Environment, vol. 13, pp. 234-246, Jan. 2019, doi: 10.1016/j.rsase.2018.11.001.

[25] A. Senta and L. Šerić, "Remote sensing data driven bathing water quality assessment using sentinel-3," Indonesian Journal of Electrical Engineering and Computer Science (IJEECS), vol. 21, no. 3, pp. 1634-1647, Mar. 2021, doi: 10.11591/ijeecs.v21.i3.pp1634-1647.

[26] N. Yaa'cob, M. F. Ayob, N. Tajudin, M. Kassim, and A. L. Yusof, "Single event latch-up detection for nano-satellite external solar radiation mitigation system," Bulletin of Electrical Engineering and Informatics (BEEI), vol. 10, no. 1, pp. 39-45, Feb. 2021, doi: 10.11591/eei.v10i1.2488.

[27] D. Ienco, R. Gaetano, P. Maurel, and D. Ienco, "Land cover classification via multitemporal spatial data by deep recurrent neural networks (Ienco17) intro to satellite image time series (SITS) data," IEEE Geoscience and Remote Sensing Letters, vol. 14, no. 10, pp. 1685-1689, 2017.

[28] A. Roy and A. B. Inamdar, "Multi-temporal land use land cover (LULC) change analysis of a dry semi-arid river basin in western India following a robust multi-sensor satellite image calibration strategy," Heliyon, vol. 5, no. 4, Apr. 2019, Art. no. e01478, doi: 10.1016/j.heliyon.2019.e01478. 


\section{BIOGRAPHIES OF AUTHORS}

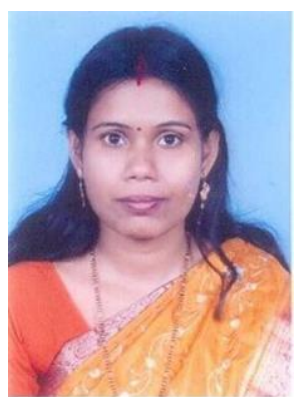

Subhra Swetanisha (D) 8 SC P has been working as an Assistant Professor in the department of Computer Science and Engineering at Trident Academy of Technology, Bhubaneswar, Odisha. She has completed M.Tech. degree in Computer Science and Engineering from KIIT Deemed to be University, Bhubaneswar and also continuing her Ph.D. Her research interests include Machine Learning, Data Science, Image Processing and Remote Sensing. She has sixteen years of teaching experience and published more than ten Scopus/SCI indexed research articles. She is a life member of the ISTE and IAENG. She can be contacted at email: sswetanisha@gmail.com.

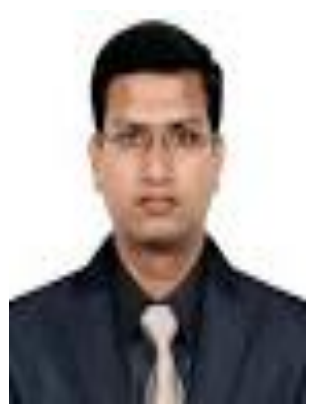

Amiya Ranjan Panda (D) SC P has seven years of research experience in DRDO and more than three years of teaching experience. He received B.Tech. degree in Information Technology from Biju Patnaik University of Technology, Rourkela, India, in 2009, and the M.Tech. degree in Computer Science and Engineering from the Kalinga Institute of Industrial Technology (KIIT), Bhubaneshwar, India, in 2012. He received Ph.D. degree from Siksha 'O' Anusandhan University, Bhubaneshwar, in 2017, working in a real-time project, 'Design, development and implementation of Software Defined Radio based Flight Termination System' of DRDO, ITR, Chandipur. He has worked as JRF, SRF and RA in DRDO, ITR, Chandipur, for more than seven years. Then, he has worked as Assistant Professor at Siksha ' $\mathrm{O}$ ' Anusandhan University for four months, and currently, he is working as Assistant Professor in KIIT Deemed to be University, Bhubaneswar. His research interest is machine learning, IoT, data acquisition system and software-defined radio. He has published more than 25 articles in international journals. Currently, he is working with two nos of real-time projects of DRDO. He can be contacted at email: amiya.pandafcs@ kiit.ac.in.

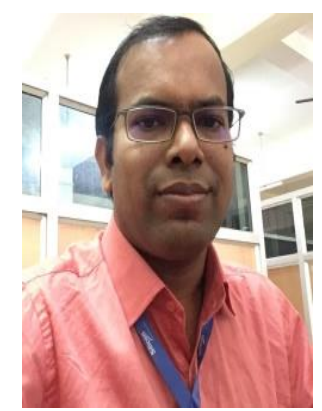

Dayal Kumar Behera (iD $8 \mathrm{SC}$ P has fifteen years of teaching experience and received Ph.D. degree from KIIT Deemed to be University. He has obtained B.E. degree with honours in Information Technology from the National Institute of Science and Technology, Berhampur, Odisha, in 2006 and completed M.Tech. from the College of Engineering and Technology Bhubaneswar in 2012. He has been working as an Assistant Professor in CSE department at Silicon Institute of Technology, Bhubaneswar. His research interest includes Recommendation Systems, Machine Learning, IoT, and Image Processing. He has fifteen publications in Scopus/SCI indexed Journals and conferences. He has guided many M.Tech. Projects and two IEDC funded projects in his area of interest. He is a lifetime member of ISTE and IAENG societies. He can be contacted at email: dayalbehera@gmail.com. 